\title{
Automated Search for Flare Stars Continued
}

\author{
J. Winterberg ${ }^{1}$, M. Nolte ${ }^{1}$, W. C. Seitter ${ }^{1}, H$. W. Duerbeck ${ }^{1}$ \\ M. K. Tsvetkov ${ }^{2}$, K. P. Tsvetkova ${ }^{2}$ \\ 1 Astronomical Institute of Muenster (AIM), Germany \\ 2 Institute of Astronomy, Bulgarian Academy of Sciences, Sofia, Bulgaria
}

\section{Introduction}

To date we obtained 511 blue plates with multiple exposures of nine young clusters and associations to be used for studying the flare activity-age relation. First attempts showed already that automatic search increases the number of discovered flares by $50 \%$ compared to visual inspection (Aniol et al. 1989). Improved algorithms have now been implemented and applied. Automated object identification in existing catalogues (FSDB, GCVS, SIMBAD) is also available.

\section{Reductions}

All plates are digitized with the PDS $2020 \mathrm{GM}^{\text {plus }}$ microdensitometers at AIM and reduced with the AIM software. Steps following the reconstruction of images include the transformation of plate to sky coordinates and matching of the multiple exposure chains with the stars on the deep reference plate. All complete chains (generally six exposures) are used to derive average light curves for all objects. For each magnitude interval $10-11,11-12, \ldots 15-16$ the mean deviation and standard error of an image from the overall mean value of the respective chains is determined. If any image in a chain differs by more than a preset multiple of the mean error it is considered a potential flare event and registered for visual inspection, together with the chains immediately preceding and following the suspected flare event, to obtain information on the total duration of the outburst. The candidates are filed and the data of all stars are stored for checks of long-term variability.

\section{Results in the Orion Region}

The stellar aggregate M42/M43 in Orion is the region best observed for flare stars (Tsvetkova et al. 1995). In our campaign from 1985 to 1990 we obtained 53 blue plates with a total of 59 hours observing time at the GPO astrograph at ESO/La Silla. 23 flares were observed, examples of two slow flares are are given in Figure 2, 5 flare were found on hitherto unknown flare stars. 


\section{Statistics}

The detection rate on GPO astrograph plates, using automated search procedures, is 0.10 flares per hour and square degree. The mean rate of six large published investigations is $0.06 \pm 0.02$ in the same units. This shows that astrographs, in spite of missing the bright ultraviolet contributions, together with automated search procedures are suitable tools for flare detection.

Another aspect of automated search of flare stars are the types of flares registered. A test between visual inspection of plates by an experienced observer showed that an extremely short bright event was only registered by eye. Otherwise, as might be expected, our automatic procedure found more slow flares and flares on bright stars than detected by eye. This is supported by the fact that on all plates reduced so far, 4 of our 23 detected flares in Orion had rising times $>20$ min in comparison with only 25 slow flare events registered in the region up to 1993 (Parsamian et al. 1993).

Some of the flares occurred in known In- and Ins-type variable stars. Their variations are visible in the long term light curves derived from all exposures (see also Aniol et al. 1989).

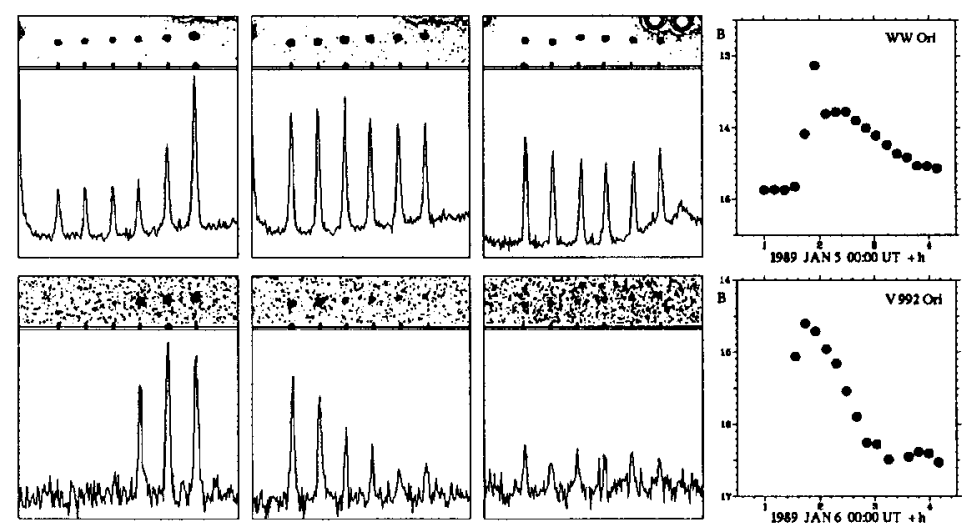

Fig. 1. Light curves of flares in WW Ori and V992 Ori. Isopleths from the plates (upper part left) and tracings (lower part left) are shown together with the reconstructed, calibrated light curves (right).

Acknowledgements: M.K.T. and K.P.T. thank the DFG and BNSF (project F340/93) for support, J.W. acknowledges support from the IAU.

\section{References}

Aniol R. et al., 1989, IAU Symp. 137, p. 85

Parsamian E.S., Chavira E., Gonzalez G., 1993, Rev. Mex. Astron. Astrof. 25, 71

Tsvetkova K.P. et al., 1995, this volume, p. 121 